\title{
On the Duality between MIMO Systems with Distributed Antennas and MIMO Systems with Colocated Antennas
}

\author{
Jan Mietzner ${ }^{1}$ and Peter A. Hoeher ${ }^{2}$ \\ ${ }^{1}$ Communication Theory Group, Department of Electrical and Computer Engineering, The University of British Columbia, \\ 2332 Main Mall, Vancouver, BC, Canada V6T $1 Z 4$ \\ ${ }^{2}$ Information and Coding Theory Lab, Faculty of Engineering, University of Kiel, Kaiserstrasse 2, 24143 Kiel, Germany
}

Correspondence should be addressed to Jan Mietzner, janm@ece.ubc.ca

Received 1 May 2007; Revised 16 August 2007; Accepted 28 October 2007

Recommended by M. Chakraborty

\begin{abstract}
Multiple-input multiple-output (MIMO) systems are known to offer huge advantages over single-antenna systems, both with regard to capacity and error performance. Usually, quite restrictive assumptions are made in the literature on MIMO systems concerning the spacing of the individual antenna elements. On the one hand, it is typically assumed that the antenna elements at transmitter and receiver are colocated, that is, they belong to some sort of antenna array. On the other hand, it is often assumed that the antenna spacings are sufficiently large, so as to justify the assumption of uncorrelated fading on the individual transmission links. From numerous publications it is known that spatially correlated links caused by insufficient antenna spacings lead to a loss in capacity and error performance. We show that this is also the case when the individual transmit or receive antennas are spatially distributed on a large scale, which is caused by unequal average signal-to-noise ratios (SNRs) on the individual transmission links. Possible applications include simulcast networks as well as future mobile radio systems with joint transmission or reception strategies. Specifically, it is shown that there is a strong duality between MIMO systems with colocated antennas (and spatially correlated links) and MIMO system with distributed antennas (and unequal average link SNRs). As a result, MIMO systems with distributed and colocated antennas can be treated in a single, unifying framework. An important implication of this finding is that optimal transmit power allocation strategies developed for MIMO systems with colocated antennas may be reused for MIMO systems with distributed antennas, and vice versa.
\end{abstract}

Copyright (c) 2008 J. Mietzner and P. A. Hoeher. This is an open access article distributed under the Creative Commons Attribution License, which permits unrestricted use, distribution, and reproduction in any medium, provided the original work is properly cited.

\section{INTRODUCTION}

Multiple-input multiple-output (MIMO) systems have gained much attention during the last decade, because they offer huge advantages over conventional single-antenna systems. On the one hand, it was shown in [1-3] that the capacity of a MIMO system with $M$ transmit (Tx) antennas and $N$ receive $(\mathrm{Rx})$ antennas grows linearly with $\min \{M, N\}$. Correspondingly, multiple antennas provide an excellent means to increase the spectral efficiency of a system. On the other hand, it was shown in [4-6] that multiple antennas can also be utilized, in order to provide a spatial diversity gain and thus to improve the error performance of a system.

The results in [1-6] are based on quite restrictive assumptions with regard to the antenna spacings at transmitter and receiver. On the one hand, it is assumed that the individual antenna elements are colocated, that is, they are part of some antenna array (cf. Figure 1(a)). On the other hand, the antenna spacings are assumed to be sufficiently large, so as to justify the assumption of independent fading on the individual transmission links. In numerous publications, it was shown that spatial fading correlations, caused by insufficient antenna spacings (cf. Figure 1(b)), can lead to significant degradations in capacity and error performance, for example, [7-9]. In this paper, we show that this is also the case when the individual transmit and/or receive antennas are distributed on a large scale (cf. Figure 1(c)), since the individual transmission links are typically characterized by unequal average signal-to-noise ratios (SNRs) caused by unequal link lengths and shadowing effects. Application examples include simulcast networks for broadcasting or paging applications, where multiple distributed transmitting nodes 


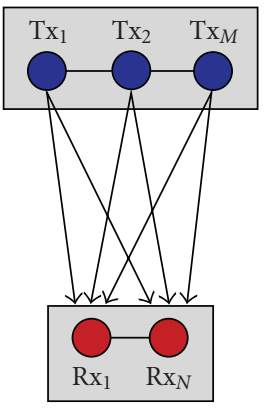

(a)

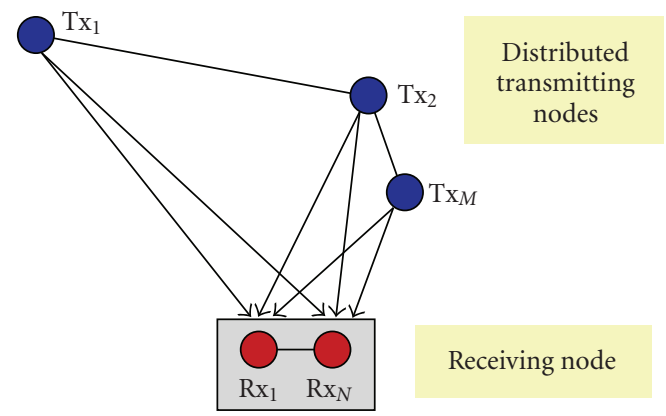

(c)

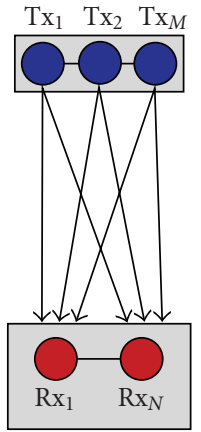

(b)
FIGURE 1: MIMO systems with different antenna spacings, for the example of $M=3$ transmit and $N=2$ receive antennas: (a) MIMO system with colocated antennas (statistically independent links); (b) MIMO system with colocated antennas and insufficient antenna spacings at the transmitter side (spatially correlated links); (c) MIMO system with distributed antennas at the transmitter side (and unequal average link SNRs).

(typically base stations) serve a common geographical area by performing a joint transmission strategy [10], as well as future mobile radio systems, where joint transmission and reception strategies among distributed wireless access points are envisioned [11]. In particular, we show that there is a strong duality between MIMO systems with colocated antennas (and spatially correlated links) and MIMO systems with distributed antennas (and unequal average link SNRs). To this end, we will consider resulting capacity distributions as well as the error probabilities of space-time codes. An important implication of the above duality is that optimal transmit power allocation strategies developed for MIMO systems with colocated antennas can be reused for MIMO systems with distributed antennas, and vice versa.

In practice, there are several important differences between MIMO systems with colocated antennas and MIMO systems with distributed antennas. For example, synchronization issues are typically more crucial when antennas are spatially distributed. Furthermore, in a scenario with distributed antennas, the exchange of transmitted/received messages between the individual antenna elements may entail error-propagation effects. In order to establish a strict duality between colocated and distributed antennas, we will employ a somewhat simplified common framework here. In particular, we will assume that perfect synchronization and an error- free exchange of messages between distributed antennas are possible. (In the application examples mentioned above, the exchange of messages can, for example, be performed via some fixed backbone network, possibly by employing some error-detecting channel code.) Thus, our simplified framework yields ultimate performance limits for MIMO systems with distributed antennas. Still, the major finding of this paper, namely that MIMO systems with distributed antennas and unequal average link SNRs behave in a very similar way as MIMO systems with correlated antennas (with regard to various performance measures) will also be valid if the assumptions of perfect synchronization and error-free message exchange between distributed antennas are dropped.

The duality results presented here are based on two unitary matrix transforms. The first transform associates a given MIMO system with colocated antennas with a corresponding MIMO system with distributed antennas. This transform is related to the well-known Karhunen-Loève transform [12, Chapter 8.5], which is often used in the literature, in order to analyze correlated systems. Moreover, we introduce a second transform which associates a given MIMO system with distributed antennas with a corresponding MIMO system with colocated antennas. Although the performance of MIMO systems with distributed antennas has already been considered in various publications, mainly with focus on cooperative relaying systems, for example, [13-19], the impact of unequal average link SNRs, and particularly its close relation to spatial correlation effects, has not yet been clearly formulated in the literature. In fact, some papers on cooperative relaying neglect the impact of unequal average link SNRs completely; for example, see [13].

\subsection{Remark on spatially correlated MIMO systems}

When referring to spatial fading correlations, the notion of "insufficient antenna spacings" is somewhat relative, because spatial correlation effects are not only governed by the geometry of the antenna array and the employed carrier frequency, but also by the richness of scattering from the physical environment and the angular power distribution of the transmitted/received signals [7-9]. In cellular radio systems with a typical urban environment, for example, antenna correlations are thus observed both at the base stations (since the transmitted/received signals are typically confined to comparatively small angular regions) and at the mobile terminals (since antenna spacings are typically rather small). Even in rich-scattering (e.g., indoor) environments, where spatial correlation functions typically decay quite fast with growing antenna spacings, there are usually pronounced side lobes within the spatial correlation functions, so that unfavorable antenna spacings can still entail notable spatial correlations.

\subsection{Paper organization}

The paper is organized as follows. First, the system and correlation model used throughout this paper are introduced in Section 2. Then, the duality between MIMO systems with distributed antennas and MIMO systems with colocated antennas is established in Section 3, with regard to the resulting 
capacity distribution (Section 3.1), the pairwise error probability of a general space-time code (Section 3.2), and the symbol error probability of an orthogonal space-time block code (OSTBC) [5, 6] (Section 3.3). The most important results are summarized in Theorems 1-3. Finally, optimal transmit power allocation strategies for MIMO systems with colocated and distributed antennas are briefly discussed in Section 4, and some conclusions are offered in Section 5.

\subsection{Mathematical notation}

Matrices and vectors are written in upper case and lower case bold face, respectively. If not stated otherwise, all vectors are column vectors. The complex conjugate of a complex number $a$ is marked as $a^{*}$ and the Hermitian transposed of a matrix $\mathbf{A}$ as $\mathbf{A}^{\mathrm{H}}$. The $(i, j)$ th element of $\mathbf{A}$ is denoted as $a_{i j}$ or $[\mathbf{A}]_{i, j}$. The trace and the determinant of $\mathbf{A}$ are denoted as $\operatorname{tr}(\mathbf{A})$ and $\operatorname{det}(\mathbf{A})$, respectively. Moreover, $\|\mathbf{A}\|_{F}=\sqrt{\operatorname{tr}\left(\mathbf{A A}^{\mathrm{H}}\right)}$ denotes the Frobenius norm of $\mathbf{A}, \operatorname{diag}(\mathbf{a})$ a diagonal matrix with diagonal elements given by the vector $\mathbf{a}$, and $\operatorname{vec}(\mathbf{A})$ a vector which results from stacking the columns of matrix $\mathbf{A}$ in a single vector. Finally, $\mathbf{I}_{n}$ denotes the $(n \times n)$-identity matrix, $\mathrm{E}\{\cdot\}$ denotes statistical expectation, and $\delta\left[k-k_{0}\right]$ denotes a discrete Dirac impulse at $k=k_{0}$.

\section{SYSTEM AND CORRELATION MODEL}

Throughout this paper, complex baseband notation is used. We consider a point-to-point MIMO communication link with $M$ transmit and $N$ receive antennas. The antennas are either colocated or distributed and are assumed to have fixed positions. The discrete-time channel model for quasi-static frequency-flat fading is given by

$$
\mathbf{y}[k]=\mathbf{H x}[k]+\mathbf{n}[k]
$$

where $k$ denotes the discrete time index, $\mathbf{y}[k]$ the $k$ th received vector, $\mathbf{H}$ the $(N \times M)$-channel matrix, $\mathbf{x}[k]$ the $k$ th transmitted vector, and $\mathbf{n}[k]$ the $k$ th additive noise vector. It is assumed that $\mathbf{H}, \mathbf{x}[k]$, and $\mathbf{n}[k]$ are statistically independent. The channel matrix $\mathbf{H}$ is assumed to be constant over an entire data block of length $N_{\mathrm{b}}$, and changes randomly from one data block to the next. Correspondingly, we will sometimes use the following block transmission model:

$$
\mathbf{Y}=\mathbf{H X}+\mathbf{N}
$$

where $\mathbf{Y}:=\left[\mathbf{y}[0], \ldots, \mathbf{y}\left[N_{\mathrm{b}}-1\right]\right], \mathbf{X}:=\left[\mathbf{x}[0], \ldots, \mathbf{x}\left[N_{\mathrm{b}}-1\right]\right]$, and $\mathbf{N}:=\left[\mathbf{n}[0], \ldots, \mathbf{n}\left[N_{\mathrm{b}}-1\right]\right]$. The entries $h_{j i}$ of $\mathbf{H}(i=$ $1, \ldots, M, j=1, \ldots, N)$ are assumed to be zero-mean, circularly symmetric complex Gaussian random variables with variance $\sigma_{j i}^{2} / 2$ per real dimension, that is, $h_{j i} \sim \mathcal{C} \mathcal{N}\left\{0, \sigma_{j i}^{2}\right\}$ (Rayleigh fading). The instantaneous realizations of the channel matrix $\mathbf{H}$ are assumed to be perfectly known at the receiver. The covariance between two channel coefficients $h_{j i}$ and $h_{j^{\prime} i^{\prime}}$ is denoted as $\sigma_{i j, i^{\prime} j^{\prime}}^{2}:=\mathrm{E}\left\{h_{j i} h_{j^{\prime} i^{\prime}}^{*}\right\}$ and the corresponding spatial correlation as $\rho_{i j, i^{\prime} j^{\prime}}:=\sigma_{i j, i^{\prime} j^{\prime}}^{2} /\left(\sigma_{j i} \sigma_{j^{\prime} i^{\prime}}\right)$. The entries $x_{i}[k](i=1, \ldots, M)$ of the transmitted vector $\mathbf{x}[k]$ are treated as zero-mean random variables with variance $\sigma_{x_{i}}^{2}$.
Possibly, they are correlated due to some underlying spacetime code. We assume an overall transmit power constraint of $P$, that is, $\sum_{i} \sigma_{x_{i}}^{2} \leq P$. For the time being, we focus on the case of equal power allocation among the individual transmit antennas, that is, $\sigma_{x_{i}}^{2}=P / M$ for all indices $i$. Finally, the entries of $\mathbf{n}[k]$ are assumed to be zero-mean, spatially and temporally white complex Gaussian random variables with variance $\sigma_{\mathrm{n}}^{2} / 2$ per real dimension, that is, $n_{j}[k] \sim \mathcal{C} \mathcal{N}\left\{0, \sigma_{\mathrm{n}}^{2}\right\}$ for all indices $j$ and $\mathrm{E}\left\{\mathbf{n}[k] \mathbf{n}^{H}\left[k^{\prime}\right]\right\}=\sigma_{\mathrm{n}}^{2} \cdot \delta\left[k-k^{\prime}\right] \cdot \mathbf{I}_{N}$.

\subsection{MIMO systems with colocated antennas}

In the case of colocated antennas (both at the transmitter and the receiver side), all links experience on average similar propagation conditions. It is therefore reasonable to assume that the variance of the channel coefficients $h_{j i}$ is the same for all transmission links. Correspondingly, we define $\sigma_{j i}^{2}:=\sigma^{2}$ for all indices $i, j$. (A generalization to unequal variances is straightforward.) Moreover, we define

$$
\mathbf{R}_{\mathrm{Tx}}:=\frac{\mathrm{E}\left\{\mathbf{H}^{\mathrm{H}} \mathbf{H}\right\}}{\left(N \sigma^{2}\right)}, \quad \mathbf{R}_{\mathrm{Rx}}:=\frac{\mathrm{E}\left\{\mathbf{H} \mathbf{H}^{\mathrm{H}}\right\}}{\left(M \sigma^{2}\right)},
$$

where $\mathbf{R}_{\mathrm{Tx}}$ denotes the transmitter correlation matrix and $\mathbf{R}_{\mathrm{Rx}}$ the receiver correlation matrix (with $\operatorname{tr}\left(\mathbf{R}_{\mathrm{Tx}}\right):=M$ and $\left.\operatorname{tr}\left(\mathbf{R}_{\mathrm{Rx}}\right):=N\right)$. Throughout this paper, the so-called Kronecker-correlation model ${ }^{1}$ [7] is employed, that is, the overall spatial correlation matrix $\mathbf{R}:=\mathrm{E}\left\{\operatorname{vec}(\mathbf{H}) \operatorname{vec}(\mathbf{H})^{\mathrm{H}}\right\} / \sigma^{2}$ can be written as the Kronecker product

$$
\begin{gathered}
\mathbf{R}=\mathbf{R}_{\mathrm{Tx}} \otimes \mathbf{R}_{\mathrm{Rx}}, \\
\mathbf{R}_{\mathrm{Tx}}:=\left[\rho_{\mathrm{Tx}, i i^{\prime}}\right]_{i, i^{\prime}=1, \ldots, M}, \quad \mathbf{R}_{\mathrm{Rx}}:=\left[\rho_{\mathrm{Rx}, j j^{\prime}}\right]_{j, j^{\prime}=1, \ldots, N},
\end{gathered}
$$

and the channel matrix $\mathbf{H}$ can be written as

$$
\mathbf{H}:=\mathbf{R}_{\mathrm{Rx}}^{1 / 2} \mathbf{G R}_{\mathrm{Tx}}^{1 / 2}
$$

where $\mathbf{G}$ denotes an $(N \times M)$-matrix with independent and identically distributed (i.i.d.) entries $g_{j i} \sim \mathcal{C} \mathcal{N}\left\{0, \sigma^{2}\right\}$. The eigenvalue decompositions of $\mathbf{R}_{\mathrm{Tx}}$ and $\mathbf{R}_{\mathrm{Rx}}$ are in the sequel denoted as

$$
\mathbf{R}_{\mathrm{Tx}}:=\mathbf{U}_{\mathrm{Tx}} \Lambda_{\mathrm{Tx}} \mathbf{U}_{\mathrm{TX}}^{\mathrm{H}}, \quad \mathbf{R}_{\mathrm{Rx}}:=\mathbf{U}_{\mathrm{Rx}} \Lambda_{\mathrm{Rx}} \mathbf{U}_{\mathrm{Rx}}^{\mathrm{H}},
$$

where $\mathrm{U}_{\mathrm{Tx}}, \mathrm{U}_{\mathrm{Rx}}$ are unitary matrices and $\Lambda_{\mathrm{Tx}}, \Lambda_{\mathrm{Rx}}$ are diagonal matrices containing the eigenvalues $\lambda_{\mathrm{Tx}, i}$ and $\lambda_{\mathrm{Rx}, j}$ of $\mathbf{R}_{\mathrm{Tx}}$ and $\mathbf{R}_{\mathrm{Rx}}$, respectively.

\subsection{MIMO systems with distributed antennas}

Consider first a MIMO system with distributed antennas at the transmitter side. As a generalization to Figure 1(c), the individual transmitting nodes may in the sequel be equipped

\footnotetext{
${ }^{1}$ Although the Kronecker-correlation model is not the most general correlation model, it was shown to be quite accurate as long as a moderate number of transmit and receive antennas are used [20].
} 
with multiple antennas. To this end, let $T$ denote the number of transmitting nodes, $M_{t}$ the number of antennas employed at the $t$ th transmitting node $(1 \leq t \leq T)$, and let $M$ again denote the overall number of transmit antennas, that is, $\sum_{t} M_{t}=: M$. As earlier, let $N$ denote the number of receive antennas used. For simplicity, we assume that all transmit antennas are uncorrelated. (For antennas belonging to different transmitting nodes, this assumption is surely met.) A generalization to the case of correlated transmit antennas is, however, straightforward.

Similar to Section 2.1, it is again reasonable to assume that all channel coefficients associated with the same transmitting node $t$ have the same variance $\sigma_{t}^{2}$. Correspondingly, we obtain

$$
\frac{E\left\{\mathbf{H}^{\mathrm{H}} \mathbf{H}\right\}}{N}=\operatorname{diag}\left(\left[\sigma_{1}^{2}, \ldots, \sigma_{t}^{2}, \ldots, \sigma_{T}^{2}\right]\right)=: \boldsymbol{\Sigma}_{\mathrm{Tx}},
$$

where each variance $\sigma_{t}^{2}$ occurs $M_{t}$ times. Following the Kronecker-correlation model, we may thus write

$$
\mathbf{H}:=\mathbf{R}_{\mathrm{Rx}}^{1 / 2} \mathbf{G} \boldsymbol{\Sigma}_{\mathrm{Tx}}^{1 / 2},
$$

where the i.i.d. entries of $\mathbf{G}$ have variance one. Due to different link lengths (and, possibly, additional shadowing effects), the variances $\sigma_{t}^{2}$ will typically vary significantly from one transmitting node to another, since the received power decays at least with the square of the link length [21, Chapter $1.2]$.

Similarly, in the case of colocated transmit antennas and distributed receive antennas, where $R$ denotes the number of receiving nodes, we obtain

$$
\frac{\mathrm{E}\left\{\mathbf{H H}^{\mathrm{H}}\right\}}{M}=\operatorname{diag}\left(\left[\sigma_{1}^{2}, \ldots, \sigma_{r}^{2}, \ldots, \sigma_{R}^{2}\right]\right)=: \boldsymbol{\Sigma}_{\mathrm{Rx}},
$$

that is, $\mathbf{H}:=\Sigma_{\mathrm{Rx}}^{1 / 2} \mathbf{G R}_{\mathrm{Tx}}^{1 / 2}$.

\subsection{Normalization}

In order to treat MIMO systems with colocated antennas and MIMO systems with distributed antennas in a single, unifying framework, we employ the following normalization:

$$
\operatorname{tr}\left(\mathrm{E}\left\{\operatorname{vec}(\mathbf{H}) \operatorname{vec}(\mathbf{H})^{\mathrm{H}}\right\}\right):=M N .
$$

For MIMO systems with colocated antennas this means we set $\sigma^{2}:=1$. For MIMO systems with distributed transmit or receive antennas, it means we set $\operatorname{tr}\left(\boldsymbol{\Sigma}_{\mathrm{Tx}}\right):=M$ or $\operatorname{tr}\left(\boldsymbol{\Sigma}_{\mathrm{Rx}}\right):=$ $N$.

\section{DUALITY BETWEEN DISTRIBUTED AND COLOCATED ANTENNAS}

In the following, we will show that, based on the above framework, for any MIMO system with colocated antennas, which follows the Kronecker-correlation model (5), an equivalent MIMO system with distributed antennas (and unequal average link SNRs) can be found, and vice versa, in the sense that both systems are characterized by identical capacity distributions.

\subsection{Duality with regard to capacity distribution}

For the time being, we assume that no channel state information is available at the transmitter side. In this case, the (instantaneous) capacity of the MIMO system (1) is given by the well-known expression [2]

$$
C(\mathbf{H})=\log _{2} \operatorname{det}\left(\mathbf{I}_{N}+\frac{P}{M \sigma_{\mathrm{n}}^{2}} \mathbf{H} \mathbf{H}^{\mathrm{H}}\right) \text { bit/channel use, }
$$

where $C(\mathbf{H})=: r$ is a random variable with probability density function (PDF) denoted as $p(r)$.

\subsubsection{Capacity distribution for MIMO systems with colocated antennas}

The characteristic function of the instantaneous capacity, $\mathrm{cf}_{r}(\mathrm{j} \omega):=\mathrm{E}\left\{\mathrm{e}^{\mathrm{j} \omega r}\right\}(\mathrm{j}=\sqrt{-1}, \omega \in \mathbb{R})$, was evaluated in [22]. The result is of form

$$
\mathrm{cf}_{r}(\mathrm{j} \omega)=\frac{K \varphi(\mathrm{j} \omega)}{\psi\left(\mathbf{R}_{\mathrm{A}}, \mathbf{R}_{\mathrm{B}}\right)} \operatorname{det}\left(\left[\begin{array}{c}
\mathbf{V}\left(\mathbf{R}_{\mathrm{B}}\right) \\
\mathbf{M}\left(\mathbf{R}_{\mathrm{A}}, \mathbf{R}_{\mathrm{B}}, \mathrm{j} \omega\right)
\end{array}\right]\right)
$$

(see [22] for further details ${ }^{2}$, where

$$
\mathbf{R}_{\mathrm{A}}, \mathbf{R}_{\mathrm{B}}:= \begin{cases}\mathbf{R}_{\mathrm{Tx}}, \mathbf{R}_{\mathrm{Rx}} & \text { if } M<N, \\ \mathbf{R}_{\mathrm{Rx}}, \mathbf{R}_{\mathrm{Tx}} & \text { else. }\end{cases}
$$

Interestingly, the scalar term $\psi\left(\mathbf{R}_{\mathrm{A}}, \mathbf{R}_{\mathrm{B}}\right)$ as well as the Vandermonde matrix $\mathbf{V}\left(\mathbf{R}_{\mathrm{B}}\right)$ and the matrix $\mathbf{M}\left(\mathbf{R}_{\mathrm{A}}, \mathbf{R}_{\mathrm{B}}, j \omega\right)$ depend solely on the eigenvalues of $\mathbf{R}_{A}$ and $\mathbf{R}_{B}$, but not on specific entries of $\mathbf{R}_{\mathrm{A}}$ or $\mathbf{R}_{\mathrm{B}}$. Moreover, the terms $K$ and $\varphi(\mathrm{j} \omega)$ are independent of $\mathbf{R}_{\mathrm{A}}$ and $\mathbf{R}_{\mathrm{B}}$. The characteristic function $\mathrm{cf}_{r}(\mathrm{j} \omega)$ contains the complete information about the statistical properties of $r=C(\mathbf{H})$. Specifically, the PDF of $r$ can be calculated as [23, Chapter 1.1]

$$
p(r)=\frac{1}{2 \pi} \int_{-\infty}^{+\infty} \mathrm{cf}_{r}(\mathrm{j} \omega) e^{-\mathrm{j} \omega r} \mathrm{~d} \omega
$$

\subsubsection{Capacity distribution for MIMO systems with distributed antennas}

Since the characteristic function $\mathrm{cf}_{r}(\mathrm{j} \omega)$ according to (12) depends solely on the eigenvalues of $\mathbf{R}_{\mathrm{A}}$ and $\mathbf{R}_{\mathrm{B}}$, any MIMO system having an overall spatial covariance matrix

$$
\begin{aligned}
\mathrm{E}\left\{\operatorname{vec}(\mathbf{H}) \operatorname{vec}(\mathbf{H})^{\mathrm{H}}\right\} & =\left(\mathbf{U}_{M} \mathbf{R}_{\mathrm{Tx}} \mathbf{U}_{M}^{\mathrm{H}}\right) \otimes\left(\mathbf{U}_{N} \mathbf{R}_{\mathrm{Rx}} \mathbf{U}_{N}^{\mathrm{H}}\right) \\
& =: \mathbf{R}_{\mathrm{Tx}}^{\prime} \otimes \mathbf{R}_{\mathrm{Rx}}^{\prime},
\end{aligned}
$$

where $\mathbf{U}_{M}$ is an arbitrary unitary $(M \times M)$-matrix and $\mathbf{U}_{N}$ an arbitrary unitary $(N \times N)$-matrix, will exhibit exactly the same capacity distribution (14) as the above MIMO system

\footnotetext{
${ }^{2}$ For simplicity, it was assumed in [22] that both matrices $\mathbf{R}_{\mathrm{A}}$ and $\mathbf{R}_{\mathrm{B}}$ have full rank and distinct eigenvalues. If the eigenvalues of $\mathbf{R}_{\mathrm{A}}$ or $\mathbf{R}_{\mathrm{B}}$ are not distinct, the characteristic function of $r=C(\mathbf{H})$ can be obtained as a limiting case of (12).
} 
with colocated antennas (because the eigenvalues of $\mathbf{R}_{\mathrm{Tx}}^{\prime}$ and $\mathbf{R}_{\mathrm{Tx}}$ and of $\mathbf{R}_{\mathrm{Rx}}^{\prime}$ and $\mathbf{R}_{\mathrm{Rx}}$ are identical). Specifically, we may choose $\mathbf{U}_{M}:=\mathbf{U}_{\mathrm{Tx}}^{\mathrm{H}}$ and/or $\mathbf{U}_{N}:=\mathbf{U}_{\mathrm{Rx}}^{\mathrm{H}}$, in order to find an equivalent MIMO system with distributed transmit and/or distributed receive antennas:

$$
\mathbf{U}_{\mathrm{Tx}}^{\mathrm{H}} \mathbf{R}_{\mathrm{Tx}} \mathbf{U}_{\mathrm{Tx}}=\Lambda_{\mathrm{Tx}}=: \boldsymbol{\Sigma}_{\mathrm{Tx}}, \quad \mathbf{U}_{\mathrm{Rx}}^{\mathrm{H}} \mathbf{R}_{\mathrm{Rx}} \mathbf{U}_{\mathrm{Rx}}=\boldsymbol{\Lambda}_{\mathrm{Rx}}=: \boldsymbol{\Sigma}_{\mathrm{Rx}} .
$$

By this means, for any MIMO system with colocated antennas, which follows the Kronecker-correlation model, an equivalent MIMO system with distributed antennas can be found. Vice versa, given a MIMO system with distributed transmit and/or distributed receive antennas, the diagonal elements of the matrix $\boldsymbol{\Sigma}_{\mathrm{Tx}}\left(\boldsymbol{\Sigma}_{\mathrm{Rx}}\right)$, normalized according to Section 2.3, may be interpreted as the eigenvalues of a corresponding correlation matrix $\mathbf{R}_{\mathrm{Tx}}\left(\mathbf{R}_{\mathrm{Rx}}\right)$. In fact, for any number of transmit (receive) antennas, a unitary matrix $\tilde{\mathbf{U}}_{M}\left(\tilde{\mathbf{U}}_{N}\right)$ can be found such that the transform

$$
\widetilde{\mathbf{U}}_{M} \boldsymbol{\Sigma}_{\mathrm{Tx}} \widetilde{\mathbf{U}}_{M}^{\mathrm{H}}=: \mathbf{R}_{\mathrm{Tx}}, \quad \tilde{\mathbf{U}}_{N} \boldsymbol{\Sigma}_{\mathrm{Rx}} \widetilde{\mathbf{U}}_{N}^{\mathrm{H}}=: \mathbf{R}_{\mathrm{Rx}}
$$

yields a correlation matrix $\mathbf{R}_{\mathrm{Tx}}\left(\mathbf{R}_{\mathrm{Rx}}\right)$ with diagonal entries equal to one and nondiagonal entries with magnitudes $\leq 1$. Suitable unitary matrices are, for example, the $(n \times n)$-Fourier matrix with entries $\tilde{u}_{i j}=\mathrm{e}^{\mathrm{j} 2 \pi(i-1)(j-1) / n} / \sqrt{n}$ (which exists for any number $n)$, or the normalized $(n \times n)$-Hadamard matrix. Note that for $\boldsymbol{\Sigma}_{\mathrm{Tx}} \neq \mathbf{I}_{M}\left(\boldsymbol{\Sigma}_{\mathrm{Rx}} \neq \mathbf{I}_{N}\right)$, at least some nondiagonal entries of $\mathbf{R}_{\mathrm{Tx}}\left(\mathbf{R}_{\mathrm{Rx}}\right)$ in the equivalent MIMO system with colocated antennas will have magnitudes greater than zero, that is, some of the transmission links will be mutually correlated. If only a single diagonal element of $\boldsymbol{\Sigma}_{\mathrm{Tx}}\left(\boldsymbol{\Sigma}_{\mathrm{Rx}}\right)$ is unequal to zero, one obtains an equivalent MIMO system with fully correlated transmit (receive) antennas. Finally, note that within our simplified framework there is no difference between distributed and colocated antennas, as soon as $\boldsymbol{\Sigma}_{\mathrm{Tx}}=\mathbf{R}_{\mathrm{Tx}}=\mathbf{I}_{M}\left(\boldsymbol{\Sigma}_{\mathrm{Rx}}=\mathbf{R}_{\mathrm{Rx}}=\mathbf{I}_{N}\right)$.

The above findings are summarized in the following theorem.

Theorem 1. Based on the presented framework, for any MIMO system with colocated transmit and receive antennas, which is subject to frequency-flat Rayleigh fading obeying the Kronecker correlation model, an equivalent MIMO system with distributed transmit and/or distributed receive antennas (and unequal average link SNRs) can be found, and vice versa, such that both systems are characterized by identical capacity distributions.

\subsection{Duality with regard to the pairwise error probability (PEP) of space-time codes}

The results in Section 3.1 were very general and are relevant for coded MIMO systems with colocated or distributed antennas. In the following, we focus on the important class of space-time coded MIMO systems. Specifically, we will show that based on the above framework space-time coded MIMO systems with correlated antennas and space-time coded MIMO systems with distributed antennas (and unequal average link SNRs) are characterized by (asymptotically) identical pairwise error probabilities (PEPs).
To this end, consider the block transmission model (2). We assume that a space-time encoder with memory length $v$ (e.g., a space-time trellis encoder [4]) is used at the transmitter side-possibly employing distributed antennas. The space-time encoder maps a sequence of $\left(N_{b}-v\right)$ information symbols (followed by $v$ known tailing symbols) onto an $\left(M \times N_{\mathrm{b}}\right)$ space-time code matrix $\mathbf{X}\left(N_{\mathrm{b}}>M\right)$. Assuming that the channel matrix $\mathbf{H}$ is perfectly known at the receiver, the metric for maximum-likelihood sequence estimation (MLSE) reads

$$
\mu(\mathbf{Y}, \tilde{\mathbf{X}}):=\|\mathbf{Y}-\mathbf{H} \tilde{\mathbf{X}}\|_{\mathrm{F}}^{2}
$$

where $\tilde{\mathbf{X}}$ denotes a hypothesis for code matrix $\mathbf{X}$. The (average) PEP $P(\mathbf{X} \rightarrow \mathbf{E})$, that is, the probability that the MLSE decoder decides in favor of an erroneous code matrix $\mathbf{E} \neq \mathbf{X}$, although matrix $\mathbf{X}$ was transmitted, is given by [24]

$$
\begin{aligned}
P(\mathbf{X} \longrightarrow \mathbf{E}) & =\operatorname{Pr}\{\mu(\mathbf{Y}, \mathbf{E}) \leq \mu(\mathbf{Y}, \mathbf{X})\} \\
& =\mathrm{E}\left\{\mathrm{Q}\left(\sqrt{\frac{P}{2 M \sigma_{\mathrm{n}}^{2}}}\|\mathbf{H}(\mathbf{X}-\mathbf{E})\|_{\mathrm{F}}\right)\right\},
\end{aligned}
$$

where $\mathrm{Q}(x)$ denotes the Gaussian Q-function.

\subsubsection{PEP for space-time coded MIMO systems with colocated antennas}

In the sequel, we assume that the employed space-time code achieves a diversity order of $M N$ (full spatial diversity). In [24], it was shown that the PEP (19) can be expressed in the form of a single finite-range integral, according to

$$
P(\mathbf{X} \longrightarrow \mathbf{E})=\frac{1}{\pi} \int_{0}^{\pi / 2} \prod_{i=1}^{M} \prod_{j=1}^{N}\left[1+\frac{P}{4 M \sigma_{\mathrm{n}}^{2}} \frac{\xi_{\mathrm{Tx}, i} \lambda_{\mathrm{Rx}, j}}{\sin ^{2} \theta}\right]^{-1} \mathrm{~d} \theta
$$

where $\xi_{\mathrm{Tx}, 1}, \ldots, \xi_{\mathrm{Tx}, M}$ denote the eigenvalues of the matrix $(\mathbf{X}-\mathbf{E})(\mathbf{X}-\mathbf{E}){ }^{\mathrm{H}} \mathbf{R}_{\mathrm{Tx}}=: \Psi_{\mathbf{X}, \mathbf{E}} \mathbf{R}_{\mathrm{Tx}}$ and $\lambda_{\mathrm{Rx}, 1}, \ldots, \lambda_{\mathrm{Rx}, N}$ the eigenvalues of $\mathbf{R}_{\mathrm{Rx}}$, as earlier.

\subsubsection{PEP for space-time coded MIMO systems with distributed antennas}

Based on the same arguments as in Section 3.1, by evaluating (16) we can always find a MIMO system with distributed receive antennas and overall spatial covariance matrix

$$
\mathrm{E}\left\{\operatorname{vec}(\mathbf{H}) \operatorname{vec}(\mathbf{H})^{\mathrm{H}}\right\}=\mathbf{R}_{\mathrm{Tx}} \otimes \boldsymbol{\Sigma}_{\mathrm{Rx}}
$$

which leads to exactly the same PEP (20) as the above MIMO system with colocated antennas. Vice versa, given a MIMO system with distributed receive antennas, we can find an equivalent MIMO system with colocated antennas by evaluating (17). As opposed to this, a MIMO system with distributed transmit antennas and overall spatial covariance matrix

$$
\mathrm{E}\left\{\operatorname{vec}(\mathbf{H}) \operatorname{vec}(\mathbf{H})^{\mathrm{H}}\right\}=\boldsymbol{\Sigma}_{\mathrm{Tx}} \otimes \mathbf{R}_{\mathrm{Rx}}
$$


$\left(\boldsymbol{\Sigma}_{\mathrm{Tx}}:=\mathbf{U}_{\mathrm{Tx}}^{\mathrm{H}} \mathbf{R}_{\mathrm{Tx}} \mathbf{U}_{\mathrm{Tx}}\right)$ will not lead to the same PEP (20), because the eigenvalues of the matrices $\Psi_{\mathrm{X}, \mathrm{E}} \mathbf{R}_{\mathrm{Tx}}$ and $\Psi_{\mathrm{X}, \mathrm{E}} \boldsymbol{\Sigma}_{\mathrm{Tx}}$ are, in general, different. (Note that we obtain a PEP expression for space-time coded MIMO systems with distributed transmit antennas, by replacing the eigenvalues $\xi_{\mathrm{Tx}, i}$ in (20) by the eigenvalues of the matrix $\Psi_{\mathrm{X}, \mathrm{E}} \boldsymbol{\Sigma}_{\mathrm{Tx}}$.) Asymptotically, that is, for large SNR values, the PEP (20) is well approximated by [25]

$$
P(\mathbf{X} \longrightarrow \mathbf{E}) \leq\left(\frac{P}{4 M \sigma_{\mathrm{n}}^{2}}\right)^{-M N} \operatorname{det}\left(\Psi_{\mathbf{X}, \mathbf{E}} \mathbf{R}_{\mathrm{Tx}}\right)^{-N} \operatorname{det}\left(\mathbf{R}_{\mathrm{Rx}}\right)^{-M}
$$

where we have assumed that $\mathbf{R}_{\mathrm{Tx}}$ and $\mathbf{R}_{\mathrm{Rx}}$ have full rank. Since also $\Psi_{\mathrm{X}, \mathrm{E}}$ has full rank (due to the assumption that the employed space-time code achieves full spatial diversity), we obtain

$$
\begin{aligned}
\operatorname{det}\left(\boldsymbol{\Psi}_{\mathbf{X}, \mathbf{E}} \mathbf{R}_{\mathrm{Tx}}\right) & =\operatorname{det}\left(\boldsymbol{\Psi}_{\mathbf{X}, \mathbf{E}}\right) \operatorname{det}\left(\mathbf{R}_{\mathrm{Tx}}\right)=\operatorname{det}\left(\boldsymbol{\Psi}_{\mathbf{X}, \mathbf{E}}\right) \operatorname{det}\left(\boldsymbol{\Sigma}_{\mathrm{Tx}}\right) \\
& =\operatorname{det}\left(\boldsymbol{\Psi}_{\mathbf{X}, \mathbf{E}} \boldsymbol{\Sigma}_{\mathrm{TX}}\right)
\end{aligned}
$$

that is, the expression (23) does not change if $\mathbf{R}_{\mathrm{Tx}}$ is replaced by $\boldsymbol{\Sigma}_{\mathrm{Tx}}$. Therefore, asymptotically the PEP expressions for MIMO systems with distributed transmit antennas and MIMO systems with colocated transmit antennas again become the same.

The above findings are summarized in the following theorem.

Theorem 2. Based on the presented framework, for any MIMO system with colocated transmit and receive antennas, which is subject to frequency-flat Rayleigh fading obeying the Kronecker correlation model and which employs a space-time coding scheme designed to achieve full spatial diversity, an equivalent space-time coded MIMO system with distributed transmit and/or distributed receive antennas (and unequal average link SNRs) can be found, and vice versa, such that asymptotically both systems are characterized by identical average PEPs.

\subsection{Duality with regard to the symbol error probability (SEP) of OSTBCS}

In the sequel, we further specialize the above results and focus on MIMO systems that employ an orthogonal space-time block code (OSTBC) [5, 6] at the transmitter side. Based on the presented framework, it will be seen that in this case identical average symbol error probabilities (SEPs) result in MIMO systems with colocated antennas and MIMO systems with distributed antennas (for any SNR value, not only asymptotically).

\subsubsection{Average SEP for OSTBC systems with colocated antennas}

Consider again the system model (1). In the case of uncorrelated antennas, the average SEP resulting for an OSTBC system with $M$ transmit and $N$ receive antennas (employing the associated widely linear detection steps at the receiver side) can be evaluated based on an equivalent maximum-ratiocombining (MRC) system [26]

$$
z[k]=\|\mathbf{h}\|_{2} a[k]+\eta[k]
$$

with one transmit antenna and $M N$ receive antennas, where $\mathbf{h}:=\operatorname{vec}(\mathbf{H})$, cf. (1), and $\eta[k] \sim \mathcal{C} \mathcal{N}\left\{0, \sigma_{\mathrm{n}}^{2}\right\}$. The transmitted data symbols $a[k]$ are i.i.d. random variables with zero mean and variance $\sigma_{a}^{2}=P /\left(M R_{\mathrm{t}}\right)$, where $R_{\mathrm{t}} \leq 1$ denotes the temporal rate of the OSTBC under consideration. Using Craig's alternative representation of the Gaussian Q-function [27], one can find closed-form expressions for the resulting average SEP, which are in the form of finite-range integrals over elementary functions [28]. For example, in the case of a $Q-$ ary phase-shift keying (PSK) signal constellation, the average SEP can be calculated as

$$
\bar{P}_{\mathrm{s}}=\frac{1}{\pi} \int_{0}^{(Q-1) \pi / Q} \prod_{\nu=1}^{M N} \frac{\sin ^{2}(\phi)}{\sin ^{2}(\phi)+\sin ^{2}(\pi / Q) \bar{\gamma}_{\nu}} \mathrm{d} \phi
$$

Here $\bar{\gamma}_{v}=\bar{\gamma}:=P /\left(M R_{\mathrm{t}} \sigma_{n}^{2}\right)$ denotes the average link SNR in the equivalent MRC system (25), where we have again employed the normalization according to Section 2.3 (i.e., $\left.\sigma^{2}:=1\right)$.

If the antennas in the OSTBC system are correlated, we have $\mathrm{E}\left\{\mathbf{h} \mathbf{h}^{\mathrm{H}}\right\}=\mathbf{R}$, cf. (4). In this case, the resulting average SEP can still be calculated based on (26), while replacing the average link SNRs $\bar{\gamma}_{\nu}$ by transformed link SNRs [29]

$$
\bar{\gamma}_{v}^{\prime}:=\frac{P \lambda_{v}}{M R_{\mathrm{t}} \sigma_{\mathrm{n}}^{2}}
$$

where $\lambda_{\nu}(\nu=1, \ldots, M N)$ denote the eigenvalues of $\mathbf{R}$. Moreover, assuming again that the OSTBC system follows the Kronecker-correlation model, the eigenvalues $\lambda_{\nu}$ are given by the pairwise products $\lambda_{\mathrm{Tx}, i} \lambda_{\mathrm{Rx}, j}(i=1, \ldots, M, j=1, \ldots, N)$ of the eigenvalues of $\mathbf{R}_{\mathrm{Tx}}$ and $\mathbf{R}_{\mathrm{Rx}}$ [30, Chapter 12.2]. Altogether, we can thus rewrite (26) according to ${ }^{3}$

$$
\begin{aligned}
\bar{P}_{\mathrm{s}}= & \frac{1}{\pi} \int_{0}^{(Q-1) \pi / Q} \prod_{i=1}^{M} \prod_{j=1}^{N} \\
& \times \frac{\sin ^{2}(\phi) M R_{\mathrm{t}} \sigma_{\mathrm{n}}^{2}}{\sin ^{2}(\phi) M R_{\mathrm{t}} \sigma_{\mathrm{n}}^{2}+\sin ^{2}(\pi / Q) P \lambda_{\mathrm{Tx}, i} \lambda_{\mathrm{Rx}, j}} \mathrm{~d} \phi .
\end{aligned}
$$

\subsubsection{Average SEP for OSTBC systems with distributed antennas}

Obviously, the complete SEP analysis for OSTBC systems with colocated antennas depends solely on the eigenvalues of $\mathbf{R}_{\mathrm{Tx}}$ and $\mathbf{R}_{\mathrm{Rx}}$. Correspondingly, it is clear that $\bar{P}_{\mathrm{s}}$ will stay exactly the same, if we replace $\mathbf{R}_{\mathrm{Tx}}$ by $\boldsymbol{\Sigma}_{\mathrm{Tx}}:=\mathbf{U}_{\mathrm{Tx}}^{H} \mathbf{R}_{\mathrm{TX}} \mathbf{U}_{\mathrm{Tx}}$ and/or $\mathbf{R}_{\mathrm{Rx}}$ by $\boldsymbol{\Sigma}_{\mathrm{Rx}}:=\mathbf{U}_{\mathrm{Rx}}^{\mathrm{H}} \mathbf{R}_{\mathrm{Rx}} \mathbf{U}_{\mathrm{Rx}}$. By this means, we have found an equivalent OSTBC system with $M$ distributed transmit

\footnotetext{
${ }^{3}$ Similar expressions can also be derived for quadrature-amplitudemodulation (QAM) and amplitude-shift keying (ASK) constellations.
} 
antennas and/or $N$ distributed receive antennas. Vice versa, given a distributed OSTBC system, we can again find an equivalent OSTBC system with colocated antennas by evaluating (17).

The above findings are summarized in the following theorem.

Theorem 3. Based on the presented framework, for any MIMO system with colocated transmit and receive antennas, which is subject to frequency-flat Rayleigh fading obeying the Kronecker correlation model and which employs an OSTBC in conjunction with the corresponding widely linear detection at the receiver, an equivalent OSTBC system with distributed transmit and/or distributed receive antennas (and unequal average link SNRs) can be found, and vice versa, such that both systems are characterized by identical average SEPS.

\subsection{Discussion}

The previous sections have shown that MIMO systems with distributed antennas and unequal average link SNRs behave in a very similar way as MIMO systems with colocated antennas and spatially correlated links (with regard to various performance measures). In other words, both effects entail very similar performance degradations. For example, spatial fading correlations (unequal average link SNRs) can lead to significantly reduced ergodic or outage capacities [7]. With regard to space-time coding, the presence of receive antenna correlations (distributed receive antennas) always degrades the resulting PEP, particularly for high SNRs. As opposed to this, the impact of transmit antenna correlations (distributed transmit antennas) depends on the employed spacetime code and the SNR regime under consideration [24]. Concerning the average SEP of OSTBCs, correlated antennas (unequal average link SNRs) always entail a performance loss [31, Chapter 3.2.5].

Note that although the assumptions within the presented framework are rather restrictive, the major finding that MIMO systems with distributed antennas and MIMO systems with colocated antennas behave in a very similar fashion will also hold, when more general scenarios are considered. For example, if error-propagation effects or nonperfect synchronization between distributed antennas come into play, distributed antennas will still behave like spatially correlated antennas. In particular, the performance degradations caused by error-propagation or non-perfect synchronization effects will simply come on the top of those caused by unequal average link SNRs, since the effects are independent of each other. Possible generalizations of the above results to frequency-selective fading channels and more general fading scenarios (e.g., Rician and Nakagami- $m$ fading) were discussed in [31, Chapter 3.3].

\section{OPTIMAL TRANSMIT POWER ALLOCATION SCHEMES}

An important implication of the above duality is that optimal transmit power allocation strategies developed for MIMO systems with colocated antennas (see, e.g., [32] for an overview) may be reused for MIMO systems with distributed antennas, and vice versa. As an example, we will focus on the use of statistical channel knowledge at the transmitter side, in terms of the transmitter correlation matrix $\mathbf{R}_{\mathrm{Tx}}$, the receiver correlation matrix $\mathbf{R}_{\mathrm{Rx}}$, and the noise variance $\sigma_{\mathrm{n}}^{2}$. Statistical channel knowledge can easily be gained in practical systems, for example offline through field measurements, ray-tracing simulations or based on physical channel models, or online based on long-term averaging of the channel coefficients [33]. Optimal statistical transmit power allocation schemes for spatially correlated MIMO systems were, for example, derived in [33-35] with regard to different optimization criteria: minimum average SEP of OSTBCs [33], minimum PEP of space-time codes [34], and maximum ergodic capacity [35]. Based on the presented framework, these optimal power allocation strategies can directly be transferred to MIMO systems with distributed antennas.

Here, we consider the optimal transmit power allocation scheme for maximizing ergodic capacity [35]. Consider again a MIMO system with colocated antennas and an overall spatial covariance matrix $\mathbf{R}=\mathbf{R}_{\mathrm{Tx}} \otimes \mathbf{R}_{\mathrm{Rx}}$. In order to maximize the ergodic capacity of the system, it was shown in [35] that the optimal strategy is to transmit in the directions of the eigenvectors of the transmitter correlation matrix $\mathbf{R}_{\mathrm{Tx}}$. To this end, the transmitted vector in (1) is premultiplied with the unitary matrix $\mathbf{U}_{\mathrm{Tx}}$ from the eigenvalue decomposition of $\mathbf{R}_{\mathrm{Tx}}$. Moreover, a diagonal weighting matrix

$$
\mathbf{W}^{1 / 2}:=\operatorname{diag}\left(\left[\sqrt{w_{1}}, \ldots, \sqrt{w_{M}}\right]\right), \operatorname{tr}(\mathbf{W}):=M,
$$

is used in order to perform the transmit power weighting among the eigenvectors of $\mathbf{R}_{\mathrm{Tx}}$. Altogether, the transmitted vector can thus be expressed as

$$
\mathbf{x}[k]:=\mathbf{U}_{\mathrm{Tx}} \mathbf{W}^{1 / 2} \mathbf{x}^{\prime}[k],
$$

where the entries of $\mathbf{x}^{\prime}[k]$ have variance $\sigma_{\mathrm{x}^{\prime}{ }_{i}}^{2}=P / M$ for all $i=$ $1, \ldots, M$. Under these premises, the instantaneous capacity (11) becomes

$$
C\left(\mathbf{H}, \mathbf{Q}_{\mathbf{x}}\right)=\log _{2} \operatorname{det}\left(\mathbf{I}_{N}+\frac{1}{\sigma_{\mathrm{n}}^{2}} \mathbf{H Q}_{\mathbf{x}} \mathbf{H}^{\mathrm{H}}\right) \text { bit/channel use, }
$$

where $\mathbf{Q}_{\mathbf{x}}:=\mathrm{E}\left\{\mathbf{x}[k] \mathbf{x}^{\mathrm{H}}[k]\right\}=P / M \cdot \mathbf{U}_{\mathrm{Tx}} \mathbf{W} \mathbf{U}_{\mathrm{Tx}}^{\mathrm{H}}$ denotes the covariance matrix of $\mathbf{x}[k]$. Unfortunately, a closed-form solution for the optimal weighting matrix $\mathbf{W}_{\text {opt }}$ maximizing the ergodic capacity $\bar{C}\left(\mathbf{Q}_{\mathbf{x}}\right):=\mathrm{E}\left\{C\left(\mathbf{H}, \mathbf{Q}_{\mathbf{x}}\right)\right\}$ is not known. The optimal power weighting results from solving the optimization problem [35]

$$
\begin{aligned}
& \operatorname{maximize} g(\mathbf{W}):=\mathrm{E}\left\{\log _{2} \operatorname{det}\left(\mathbf{I}_{N}+\sum_{i=1}^{M} \frac{w_{i} \lambda_{\mathrm{Tx}, i} \mathbf{z}_{i} \mathbf{z}_{i}^{\mathrm{H}}}{\sigma_{\mathrm{n}}^{2}}\right)\right\} \\
& \text { subject to } \operatorname{tr}(\mathbf{W}):=M, w_{i} \geq 0 \quad \forall i,
\end{aligned}
$$

where the vectors $\mathbf{z}_{i}$ are i.i.d. complex Gaussian random vectors with zero mean and covariance matrix $\boldsymbol{\Lambda}_{\mathrm{Rx}}$. Note that the optimum power weighting depends both on the eigenvalues 
of $\mathbf{R}_{\mathrm{Tx}}$ and on the eigenvalues of $\mathbf{R}_{\mathrm{Rx}}$. Based on the same arguments as in Section 3, the resulting transmit power weighting will also be optimal for a MIMO system with distributed antennas and an overall covariance matrix $\mathbf{R}=\boldsymbol{\Sigma}_{\mathrm{Tx}} \otimes \mathbf{R}_{\mathrm{Rx}}$ or $\mathbf{R}=\mathbf{R}_{\mathrm{Tx}} \otimes \boldsymbol{\Sigma}_{\mathrm{Rx}}$ with $\boldsymbol{\Sigma}_{\mathrm{Tx}}, \boldsymbol{\Sigma}_{\mathrm{Rx}}$ given by (16). In the case of distributed transmit antennas, the prefiltering matrix $\mathbf{U}_{\mathrm{Tx}}$ reduces to the identity matrix.

The expression (32) is, in general, difficult to evaluate. In the following, we will therefore employ a tight upper bound on $\bar{C}\left(\mathbf{Q}_{\mathbf{x}}\right)$, which is based on Jensen's inequality and which greatly simplifies the optimization of $\mathbf{W}$ (see [36], where the case of equal power allocation is studied). One obtains

$$
\begin{aligned}
\bar{C}\left(\mathbf{Q}_{\mathbf{x}}\right) \leq \log _{2}(1 & +\sum_{m=1}^{N_{\min }}\left(\frac{P}{M \sigma_{\mathrm{n}}^{2}}\right)^{m} m ! \sum_{i \in \ell_{m}} w_{i_{1}} \lambda_{\mathrm{Tx}, i_{1}} \cdots w_{i_{m}} \lambda_{\mathrm{Tx}, i_{m}} \\
& \left.\times \sum_{\mathbf{j} \in \mathcal{I}_{m}} \lambda_{\mathrm{Rx}, j_{1}} \cdots \lambda_{\mathrm{Rx}, j_{m}}\right)
\end{aligned}
$$

where $N_{\min }:=\min \{M, N\}$. Furthermore, $\ell_{m}$ and $g_{m}$ denote index sets defined as

$$
\begin{aligned}
& \ell_{m}:=\left\{\mathbf{i}:=\left[i_{1}, \ldots, i_{m}\right] \mid 1 \leq i_{1}<i_{2}<\cdots<i_{m} \leq M\right\} \\
& \mathscr{g}_{m}:=\left\{\mathbf{j}:=\left[j_{1}, \ldots, j_{m}\right] \mid 1 \leq j_{1}<j_{2}<\cdots<j_{m} \leq N\right\}
\end{aligned}
$$

( $\left.m \in \mathbb{Z}, 1 \leq m \leq N_{\min }\right)$. For a fixed SNR value $P /\left(M \sigma_{\mathrm{n}}^{2}\right)$, the right-hand side of (33) can now be maximized numerically in order to find the optimum power weighting matrix $\mathbf{W}_{\text {opt }}$.

As an example, we consider a MIMO system with four colocated transmit antennas and three colocated receive antennas. (Equivalently, we could again consider a corresponding MIMO system with distributed transmit and/or distributed receive antennas.) For the correlation matrices $\mathbf{R}_{\mathrm{Tx}}$ and $\mathbf{R}_{\mathrm{Rx}}$, the single-parameter correlation matrix proposed in [37] for uniform linear antenna arrays has been taken, with correlation parameters $\rho_{\mathrm{Tx}}:=0.8$ and $\rho_{\mathrm{Rx}}:=0.7$. Figure 2 displays the ergodic capacity as a function of the SNR $P /\left(M \sigma_{\mathrm{n}}^{2}\right)$ in $\mathrm{dB}$ which results in different transmit power allocation strategies. Simulative results are represented by solid lines and the corresponding analytical upper bounds are represented by dashed lines. As can be seen, compared to the case of uncorrelated antennas (dark curve, marked with " $\mathbf{x}$ ") the ergodic capacity in the case of correlated antennas and equal power allocation (dark curve, no markers) is reduced significantly, especially for large SNR values. For the light-colored curve, the transmit power weights $w_{1}, \ldots, w_{M}$ were optimized numerically, based on (33). As can be seen, compared to equal power allocation the ergodic capacity is notably improved. For SNR values smaller than $-2 \mathrm{~dB}$, the achieved ergodic capacity is even larger than in the uncorrelated case. Further numerical results not displayed in Figure 2 indicate that the knowledge of $\mathbf{R}_{\mathrm{Rx}}$ at the transmitter side is of rather little benefit. When assuming $\mathbf{R}_{\mathrm{Rx}}:=\mathbf{I}_{N}$ at the transmitter side, the resulting power allocation is still very close to the optimum.

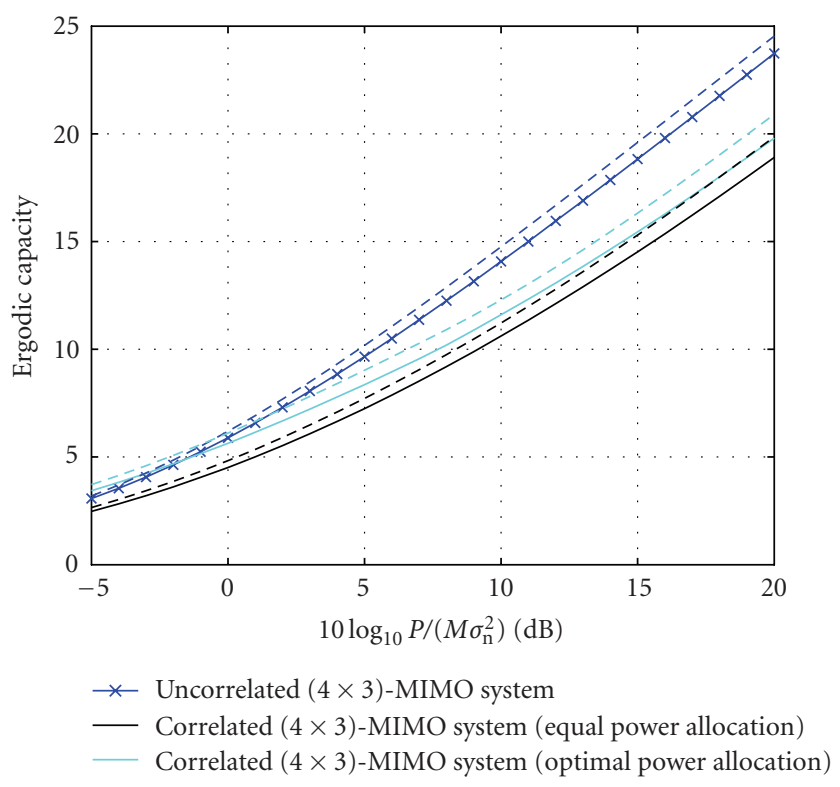

FIgure 2: Ergodic capacity as a function of the $\operatorname{SNR} P /\left(M \sigma_{\mathrm{n}}^{2}\right)$ in $\mathrm{dB}$, for different MIMO systems with $M=4$ transmit and $N=3$ receive antennas. Solid lines: simulative results obtained by means of Monte Carlo simulations over $10^{5}$ independent channel realizations. Dashed lines: corresponding analytical upper bounds based on (33).

\section{CONCLUSIONS}

In this paper, it was shown that MIMO systems with distributed antennas (and unequal average link SNRs) behave in a very similar way as MIMO systems with correlated antennas. In particular, a simple common framework for MIMO systems with colocated antennas and MIMO systems with distributed antennas was presented. Based on the common framework, it was shown that for any MIMO system with colocated antennas, which follows the Kronecker correlation model, an equivalent MIMO system with distributed antennas can be found, and vice versa, while various performance criteria were taken into account. An important implication of this finding is that optimal transmit power allocation strategies developed for MIMO systems with colocated antennas can be reused for MIMO systems with distributed antennas, and vice versa. As an example, an optimal transmit power allocation scheme based on statistical channel knowledge at the transmitter side was considered.

\section{REFERENCES}

[1] G. J. Foschini, "Layered space-time architecture for wireless communication in a fading environment when using multielement antennas," Bell Labs Technical Journal, vol. 1, no. 2, pp. 41-59, 1996.

[2] G. J. Foschini and M. J. Gans, "On limits of wireless communications in a fading environment when using multiple antennas," Wireless Personal Communications, vol. 6, no. 3, pp. 311-335, 1998.

[3] E. Telatar, "Capacity of multi-antenna Gaussian channels," European Transactions on Telecommunications, vol. 10, no. 6, pp. 585-595, 1999. 
[4] V. Tarokh, N. Seshadri, and A. R. Calderbank, "Space-time codes for high data rate wireless communication: performance criterion and code construction," IEEE Transactions on Information Theory, vol. 44, no. 2, pp. 744-765, 1998.

[5] S. M. Alamouti, "A simple transmit diversity technique for wireless communications," IEEE Journal on Selected Areas in Communications, vol. 16, no. 8, pp. 1451-1458, 1998.

[6] V. Tarokh, H. Jafarkhani, and A. R. Calderbank, "Space-time block coding for wireless communications: performance results," IEEE Journal on Selected Areas in Communications, vol. 17, no. 3, pp. 451-460, 1999.

[7] D.-S. Shiu, G. J. Foschini, M. J. Gans, and J. M. Kahn, "Fading correlation and its effect on the capacity of multielement antenna systems," IEEE Transactions on Communications, vol. 48, no. 3, pp. 502-513, 2000.

[8] D. Gesbert, M. Shafi, D.-S. Shiu, P. J. Smith, and A. Naguib, "From theory to practice: an overview of MIMO space-time coded wireless systems," IEEE Journal on Selected Areas in Communications, vol. 21, no. 3, pp. 281-302, 2003.

[9] M. K. Özdemir, E. Arvas, and H. Arslan, "Dynamics of spatial correlation and implications on MIMO systems," IEEE Communications Magazine, vol. 42, no. 6, pp. S14-S19, 2004.

[10] A. Wittneben, "Basestation modulation diversity for digital simulcast," in Proceedings of IEEE Vehicular Technology Conference (VTC '91), pp. 848-853, St. Louis, Mo, USA, May 1991.

[11] S. Zhou, M. Zhao, X. Xu, J. Wang, and Y. Yao, "Distributed wireless communication system: a new architecture for future public wireless access," IEEE Communications Magazine, vol. 41, no. 3, pp. 108-113, 2003.

[12] H. Stark and J. W. Woods, Probability and Random Processes with Applications to Signal Processing, Prentice-Hall, Upper Saddle River, NJ, USA, 3rd edition, 2002.

[13] A. Kastrisios, M. Dohler, and H. Aghvami, "Influence of channel characteristics on the performance of VAA with deployed STBCs," in Proceedings of the 57th IEEE Semiannual Vehicular Technology Conference (VTC 2003-Spring), vol. 2, pp. 11381142, Jeju, Korea, April 2003.

[14] J. N. Laneman and G. W. Wornell, "Distributed space-timecoded protocols for exploiting cooperative diversity in wireless networks," IEEE Transactions on Information Theory, vol. 49, no. 10, pp. 2415-2425, 2003.

[15] A. Sendonaris, E. Erkip, and B. Aazhang, "User cooperation diversity-part I: system description,” IEEE Transactions on Communications, vol. 51, no. 11, pp. 1927-1938, 2003.

[16] A. Sendonaris, E. Erkip, and B. Aazhang, "User cooperation diversity-part II: implementation aspects and performance analysis," IEEE Transactions on Communications, vol. 51, no. 11, pp. 1939-1948, 2003.

[17] M. Janani, A. Hedayat, T. E. Hunter, and A. Nosratinia, "Coded cooperation in wireless communications: space-time transmission and iterative decoding," IEEE Transactions on Signal Processing, vol. 52, no. 2, pp. 362-371, 2004.

[18] P. A. Anghel and M. Kaveh, "Exact symbol error probability of a cooperative network in a Rayleigh-fading environment," IEEE Transactions on Wireless Communications, vol. 3, no. 5, pp. 1416-1421, 2004.

[19] X. Li, "Space-time coded multi-transmission among distributed transmitters without perfect synchronization," IEEE Signal Processing Letters, vol. 11, no. 12, pp. 948-951, 2004.

[20] H. Ozcelik, M. Herdin, W. Weichselberger, J. Wallace, and E. Bonek, "Deficiencies of 'Kronecker' MIMO radio channel model," Electronics Letters, vol. 39, no. 16, pp. 1209-1210, 2003.
[21] R. Steele, Ed., Mobile Radio Communications, IEEE Press, New York, NY, USA, 1994.

[22] S. Park, H. Shin, and J. H. Lee, "Capacity statistics and scheduling gain for MIMO systems in correlated Rayleigh fading," in Proceedings of the 60th IEEE Vehicular Technology Conference (VTC '04), vol. 2, pp. 1508-1512, Los Angeles, Calif, USA, September 2004.

[23] J. G. Proakis, Digital Communications, McGraw-Hill, New York, NY, USA, 4th edition, 2001.

[24] J. Wang, M. K. Simon, M. P. Fitz, and K. Yao, "On the performance of space-time codes over spatially correlated Rayleigh fading channels," IEEE Transactions on Communications, vol. 52, no. 6, pp. 877-881, 2004.

[25] H. Bolcskei and A. J. Paulraj, "Performance of space-time codes in the presence of spatial fading correlation," in Proceedings of the 34th Asilomar Conference on Signals, Systems and Computers, vol. 1, pp. 687-693, Pacific Grove, Calif, USA, October-November 2000.

[26] H. Shin and J. H. Lee, "Exact symbol error probability of orthogonal space-time block codes," in Proceedings of IEEE Global Telecommunications Conference (Globecom '02), vol. 2, pp. 1197-1201, Taipei, China, November 2002.

[27] J. W. Craig, "A new, simple and exact result for calculating the probability of error for two-dimensional signal constellations," in Proceedings of IEEE Military Communications Conference (MILCOM'91), vol. 2, pp. 571-575, McLean, Va, USA, November 1991.

[28] M.-S. Alouini and A. J. Goldsmith, "A unified approach for calculating error rates of linearly modulated signals over generalized fading channels," IEEE Transactions on Communications, vol. 47, no. 9, pp. 1324-1334, 1999.

[29] X. Dong and N. C. Beaulieu, "Optimal maximal ratio combining with correlated diversity branches," IEEE Communications Letters, vol. 6, no. 1, pp. 22-24, 2002.

[30] P. Lancaster and M. Tismenetsky, The Theory of Matrices, Academic Press, New York, NY, USA, 2nd edition, 1985.

[31] J. Mietzner, "Spatial diversity in MIMO communication systems with distributed or co-located antennas," Ph.D. dissertation, Shaker, Aachen, Germany, 2007.

[32] A. Goldsmith, S. A. Jafar, N. Jindal, and S. Vishwanath, "Capacity limits of MIMO channels," IEEE Journal on Selected Areas in Communications, vol. 21, no. 5, pp. 684-702, 2003.

[33] S. Zhou and G. B. Giannakis, "Optimal transmitter eigenbeamforming and space-time block coding based on channel correlations," IEEE Transactions on Information Theory, vol. 49, no. 7, pp. 1673-1690, 2003.

[34] H. Sampath and A. Paulraj, "Linear precoding for space-time coded systems with known fading correlations," IEEE Communications Letters, vol. 6, no. 6, pp. 239-241, 2002.

[35] E. A. Jorswieck and H. Boche, "Channel capacity and capacityrange of beamforming in MIMO wireless systems under correlated fading with covariance feedback," IEEE Transactions on Wireless Communications, vol. 3, no. 5, pp. 1543-1553, 2004.

[36] H. Shin and J. H. Lee, "Capacity of multiple-antenna fading channels: spatial fading correlation, double scattering, and keyhole," IEEE Transactions on Information Theory, vol. 49, no. 10, pp. 2636-2647, 2003.

[37] A. van Zelst and J. S. Hammerschmidt, "A single coefficient spatial correlation model for multiple-input multipleoutput (MIMO) radio channels," in Proceedings of the 27th General Assembly of the International Union of Radio Science (URSI '02), pp. 1-4, Maastricht, The Netherlands, August 2002. 